\title{
LAS METODOLOGÍAS DE INNOVACIÓN PARA EL DESARROLLO DE NUEVOS PRODUCTOS: UN ENFOQUE AL CONSUMIDOR
}

Innovation Methodologies for the Development of New Products: An approach to the consumer

Samuel Joseph Alapizco Bobardel bonardel@gmail.com Gerardo Vázquez Rodríguez gerardo7vazquez@gmail.com 
Palabras clave: Design Thinking, Lean Startup, Agile, Metodologías de Innovación Keywords: Design Thinking, Lean Startup, Agile, Innovation methodologies.

Resumen:

El objetivo de este artículo es mostrar la conexión que tienen las metodologías de innovación Design Thinking, Lean Start Up y Agile para el desarrollo de nuevos productos, cuyo común denominador es la intima participación del consumidor final en cada uno de sus procesos. Tomando como referencia una investigación tipo documental, se hizo una descripción profunda de cada uno de los métodos, su alcance y objetivos para poder después llegar a definir el ciclo sobre el cual estarán interconectadas, sin dejar de lado al usuario final como el principal influenciador de cada una de las actividades que se proponen en los métodos. Es posible identificar para el desarrollo de nuevos productos, que la interrelación de las diferentes metodologías de innovación abordadas, permiten un mayor dinamismo y resultados más efectivo al momento de llegar al mercado.

\section{Abstract}

The purpose of this article is to show the connection between the innovation methodologies Design Thinking, Lean Start Up and Agile for the new product development, which have as a common factor the intimate participation of the final consumer in their processes. It was taken as a reference a documentary type research, a deep description of each of the methods, their scope and objectives were made to later define the cycle over which the interconnected is, without leaving the end user as the main one. Influencer of each of the sports that are proposed in the methods. It is possible to identify for the development of new products, that the interrelation of the different innovation methodologies addressed, allow greater dynamism and more effective results when they reach the market. 
Samuel Joseph Alapizco Bobardel

Gerardo Vázquez Rodríguez

\section{INTRODUCCIÓN}

La comunicación entre el cliente y el ejecutor del desarrollo es la clave para el éxito de cualquier producto. La capacidad de traducir correctamente los requerimientos o necesidades del mercado a especificaciones medibles es esencial en las compañías actuales. Aunado a esto, si se da una retroalimentación efectiva, durante el proceso de desarrollo, entre desarrollador y cliente, el producto no solo cumplirá con el requerimiento si no que el tiempo de desarrollo será menor y el costo de fabricarlo disminuye también. Debido a lo anterior, es relevante el involucramiento del consumidor en el proceso de desarrollo de nuevos productos, no sólo en las etapas iniciales, si no durante todas y cada una de ellas. Es el guía, consultor y auditor en el proceso de ideación, creación y producción. Su aportación efectiva asegura el éxito del producto. 


\section{LAS METODOLOGÍAS DE INNOVACIÓN}

Las compañías actuales buscan cómo hacer sus procesos de innovación utilizados para la creación de nuevos productos, desde la ideación, gestión hasta el desarrollo e implementación, con más impacto en el mercado, es decir, más efectivos en los consumidores finales.

Es por esto por lo que las metodologías de Design Thinking, Lean Startup y Agile son ampliamente utilizadas en las organizaciones a nivel mundial ya que cada una contribuye, con sus alcances, al ciclo de desarrollo de nuevos productos, sin importar la procedencia de cada una de ellas, tanto de la industria de diseño, como de los negocios, hasta el de la tecnología de información.

El modelo propuesto por Simon Mingay en la consultoría Garter, describe cómo estas 3 metodologías mencionadas interactúan de una manera tal que el proceso se puede ver de una manera continua al unir cada una de ellas, las cuales comparten la idea de que el cliente es el protagonista durante todo el proceso de desarrollo.

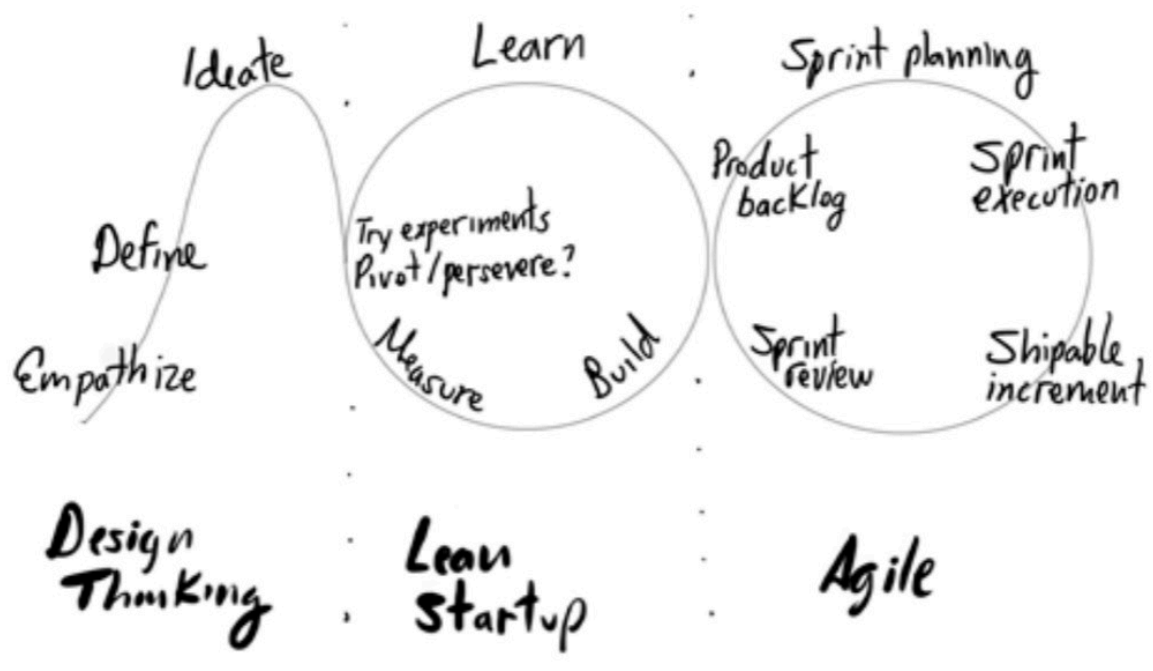

Figura 1: Combinación de los tres métodos: Design thinking, Lean Up y Agile. Elaboración: propia. 


\section{DESIGN THINKING}

En la primera etapa del ciclo de desarrollo se identifican las necesidades a cubrir o los problemas a resolver, además de validar que exista una tecnología necesaria para resolverlo. Esta fase se denomina ideación e involucra todos los procesos necesarios para definir lo que se quiere hacer mediante el uso de las herramientas del Design Thinking.

Design Thinking se define como el proceso analítico y creativo que engancha a las personas en oportunidades para experimentar, crear y prototipar modelos, obtener retroalimentación y rediseñar (Razzouk y Shute, 2012). El Design Thinking aprovecha las capacidades que tienen las personas pero que no son pasadas por alto por otras prácticas convencionales de resolución de problemas. (Brown y Katz, 2009).

Tim Brown (2009) hace énfasis en el término "las personas primero", con lo que quiere decir que cuando un diseñador desarrolla un producto, el usuario debe estar en el centro de la historia. Se debe investigar las necesidades del consumidor y diseñar enfocado en su requerimiento. Menciona también 3 elementos que se refuerzan mutuamente en cualquier programa de diseño:

Percepción. Habla de aprender acerca de las experiencias de otros. Si las personas no expresan sus necesidades, se puede observar su comportamiento para entenderlos.

Observación. Se trata de ser perspicaces al momento de analizar el comportamiento del consumidor, incluso viendo lo que no hace y escuchando lo que no dice.

Empatía. Consiste en vivir lo que el usuario está experimentando y sintiendo. Es ponerse en sus zapatos.

El proceso de Design Thinking consiste en 5 pasos fundamentales, propuesto por la universidad Hasso-Plattner Institute of Design at Stanford: Empatizar, que trata de relacionarse directamente con el usuario. Definir, Idear, Prototipar y Probar. (Dam \& Siang, 2018) 
Empatizar: consiste en entender el problema que se trata de resolver de una manera profunda y empática, sumergirse en el ambiente donde está el problema para sensibilizarse, consultar a los expertos, relacionarse con las personas involucradas, etc.

Definir: aquí se integra toda la información recabada durante la etapa de Empatía, se analiza y se definen los problemas que se han identificado.

Ideación: aquí se empiezan a generar las ideas. Se identifican las soluciones al problema establecido y se crean alternativas para ver estos problemas. Existen muchas técnicas de ideación, como son lluvia de ideas, SCAMPER, La Peor Posible Idea, etc.

Prototipo: se crean versiones del producto a menor escala y sin altos costos, para poder así investigar las soluciones generadas del problema. Esta es una fase experimental y su objetivo es identificar la mejor posible solución al problema.

Prueba: aquí se prueba el producto por completo utilizando la mejor solución generada en la etapa del prototipo. Es la etapa final, pero al ser un proceso iterativo, los resultados generados pueden ser utilizados para redefinir uno o más problemas.

Los diseñadores que trabajan con la metodología de Design Thinking buscan resoluciones a problemas y soluciones improvisadas para incluirlas en el producto que crean. Consideran, lo que se conoce como el límite, los lugares en donde las personas extremas viven diferente piensan diferente y consumen diferente. (Brown y Katz, 2009).

En esta etapa del proceso de diseño se trata de extraer los requerimientos más íntimos de los consumidores, los problemas que trata de resolver o las necesidades que requiere cubrir. Es aquí cuando se debe preguntar si se está considerando desarrollar el producto correcto. 


\section{LEAN START UP POR ERIC RICE}

Una vez identificado lo que se quiere desarrollar, es necesario convertir este producto en un modelo de negocio, el cual sea probado rápidamente con el cliente final, en el mercado real, aprender de eso y actuar en consecuencia. Este proceso se basa en la metodología Lean Start Up, propuesta por Eric Rice (2011)

Rice define esta metodología como un soporte de desarrollo de nuevos productos, de ciclos iterativos basado en aprendizajes aprendidos y construidos sobre principios esbeltos. Esta metodología prueba las ideas rápidamente con clientes con la ideología de si fracasas rápido, fracasas barato; es decir, que es mejor retrabajos en las etapas iniciales del desarrollo del producto, que en etapas más avanzadas donde los cambios cuestan más, tanto tiempo, como dinero. (Rice, 2011).

La metodología Lean Startup tiene tres principios (Blank, 2013):

- En vez de pasar meses en planeación e investigación, los emprendedores solo tienen, al primer día, una serie de hipótesis sin probar. No hacen un plan de negocio, si no que resumen sus teorías en un marco de referencia llamado lienzo de modelo de negocio.

- Buscan probar sus hipótesis directamente con el cliente, a este proceso le llaman desarrollo de consumidor. Buscan retroalimentación de los posibles compradores de todos los elementos del plan de negocios, incluyendo las características del producto, precio, canales de distribución, etc.

- Practican el desarrollo ágil, el cual se basa en el trabajo mano a mano con el consumidor en la etapa de desarrollo del producto. Con esto se busca eliminar los tiempos de desperdicio y los recursos, al hacer la metodología más iterativa. 
El éxito del método Lean Startup se basa en el circuito Crear-Medir-Aprender, que es considerado el núcleo de la metodología. La primera etapa, que es Crear, trata de transformar las ideas y desarrollar un producto, en su versión más simple, con el fin de ser probado anticipadamente por los consumidores. La segunda etapa, que es la de medición, consiste en medir las respuestas de los usuarios cuando se le presenta el producto. Por último, esa la etapa de Aprendizaje, que, en base a los resultados obtenidos en la etapa anterior, se puede tomar la decisión de seguir o de cambiar de estrategia.

En esta etapa se valida si el producto que se está proponiendo desarrollar es viable en el mercado a través de pruebas con un prototipo básico directamente con el consumidor, quien da la retroalimentación para así poder hacerle cambios al producto original. Este proceso iterativo propone asegurar la viabilidad del producto en el mercado.

\section{AGILE}

La última etapa del proceso se basa en establecer una manera sistematizada de trabajo, que se destaca por proponer diferentes iteraciones con el cliente final, en base a la muestra del producto, para poder hacer así hacer cambios o adaptar el producto propuesto en base a las retroalimentaciones recibidas. Este proceso se basa en el método Agile.

Esta metodología ha sido mayormente utilizada en aplicaciones de software, aunque últimamente ha ido tomando importancia en otras áreas de la industria. Según Thathagat Varma (2015), el término ágil se refiere a la fácil adaptación de la mente a una situación de evolución rápida y la búsqueda de maneras más eficientes para la resolución de problemas.

En 1990 una serie de metodologías de desarrollo de software evolucionaron como rebeldía por los métodos que prevalecían en aquel entonces, mismos que eran estrictos, sobre planeados, excesivamente regulados y controlados de manera cautelosa, según los críticos en la materia. Fue así como en 2001, desarrolladores de software se reunieron para discutir acerca de estas nuevas metodologías ligeras y juntos desarrollaron el “Manifiesto de Desarrollo de Software Ágil”. (Schneider, 2017) 
El manifiesto del Desarrollo de Software Agile establece que se descubren mejores maneras para desarrollar software al hacerlo y ayudando a otros a hacerlo. Se establecen los siguientes preceptos, considerando que lo que está resaltado es más valorado que los que no lo está:

- Los individuos y las interacciones sobre los procesos y las herramientas.

- Software funcionando sobre documentación de comprensión.

- La colaboración con el cliente sobre las negociaciones de contrato.

- La respuesta al cambio sobre el seguimiento a un plan.

También, en dicho manifiesto, se establecen los siguientes principios fundamentales:

- La satisfacción del cliente debido a una entrega a tiempo y continua de software valioso.

- Apertura hacia los cambios en requerimientos, aún en etapa tardía de desarrollo.

- El software trabajado es entregado frecuentemente (semanas en vez de meses).

- Una cooperación cercana y diaria entre el equipo de desarrollo y el equipo del negocio.

- Los proyectos se desarrollan alrededor de individuos motivados, a los cuales se les debe de tener confianza.

- La comunicación cara a cara es la mejor forma de comunicación.

- El software trabajado es la medida primaria de progreso.

- El desarrollo sostenible, capaz de mantener un ritmo constante. 
- Atención continua a la excelencia técnica y al buen diseño.

- Simplificación, el arte de maximizar la cantidad de trabajo no realizado, es esencial.

- Las mejores arquitecturas, requerimientos y diseños emergen de los equipos auto organizados.

- Regularmente, el equipo reflexiona en cómo ser más efectivo y se ajusta en consecuencia.

En esta etapa final del proceso de desarrollo se pregunta si se están haciendo las cosas correctamente a través de la retroalimentación constante de los clientes, la cual sirve para hacer modificaciones al producto.

\section{CONCLUSIONES}

En este análisis se evidencia la importancia de la participación del consumidor en la etapa de desarrollo de nuevos productos a través de diferentes metodologías de innovación, que en conjunto forman una sola con el fin de hacer el proceso más dinámico y efectivo al momento de llegar al mercado.

Los métodos considerados en el estudio tienen como común denominador al cliente como su eje central, siendo el protagonista en cualquier etapa de desarrollo del producto, recibiendo de él la retroalimentación necesaria para la tarea de ajustes al producto durante el ciclo de vida de desarrollo, adaptándolo a sus necesidades.

Al unir las metodologías se puede ver un modelo de desarrollo de nuevos productos integral, que considera las ventajas de cada uno de los métodos, los relaciona en las etapas que tienen en común desde la ideación hasta la implementación e incluye procesos cíclicos internos que involucran al consumidor.

Debido al origen de las metodologías utilizadas, el método propuesto puede ser utilizado para desarrollar cualquier tipo de producto o servicio, sin importar tampoco el país o el mercado en donde se vaya a aplicar. 


\section{BIBLIOGRAFÍA}

Blank, S. (2013). Why the Lean Startup Changes Everything. Harvard Buiness Review, 91(5), $1-9$.

Brown, T., y Katz, B. (2009). Change by Design: How design thinking transforms organizations and inspires innovation. New York: Harper Business.

Dam, R., y Siang, T. (2018). Stage 5 in the Design Thinking Process. Retrieved from Interacution Design Foundation. Recuperado de: https://www.interaction-design.org/literature/ article/5-stages-in-the-design-thinking-process

Razzouk, R., y Shute, S. (2012). What Is Design Thinking and Why Is It Important?. Review of Educational Research, 82(3), 330-348.

Rice, E. (2011). The Lean Startup: How Today's Entrepreneurs Use Continuous Innovation to Create Radically Successful Businesses. New York: Crown Business.

Schneider, J. (2017). Understanding Design Thinking, Lean, and Agile. Sebastopol: O’Reilly Media, Inc.

Varma, T. (2015). Agile Product Delopment: How to design innovative products that create customer value. New York: Springer. 